\title{
Metabolomics continues to expand: highlights from the 2015 metabolomics conference
}

\author{
Oliver Fiehn ${ }^{1}$ - Sastia P. Putri ${ }^{2} \cdot$ Kazuki Saito ${ }^{3,4} \cdot$ Reza M. Salek $^{5}$. \\ Darren J. Creek ${ }^{6}$
}

Published online: 13 August 2015

(c) Springer Science+Business Media New York 2015

The 11th annual international conference of the Metabolomics Society, held in San Francisco from June 29th to July 2nd 2015, lived up to its reputation as the highlight of the metabolomics calendar. With over 700 oral and poster presentations and almost 1000 attendees, this was the largest metabolomics conference to date. The conference was opened by an inspiring demonstration of the role of metabolomics in the future of personalized medicine by Mike Snyder (Stanford University). Professor Snyder described a comprehensive systems approach to personalized medicine, combining longitudinal measurements of the metabolome, genome, transcriptome, proteome and microbiome in response to lifestyle changes. The presentation gave a snapshot of the way that mature omics technologies could enable systems medicine to predict and monitor health and disease. Another highlight was the presentation of iKnife technology by Zoltan Takats (Imperial College London). This unique technology involving rapid evaporative ionization mass spectrometry coupled to an electrosurgical knife demonstrates the implementation of metabolomics-based

Darren J. Creek

darren.creek@monash.edu

1 UC Davis Genome Center, University of California Davis, Davis, CA, USA

2 Graduate School of Engineering, Osaka University, Osaka, Japan

3 RIKEN Center for Sustainable Resource Science, Yokohama, Japan

4 Chiba University, Chiba, Japan

5 European Bioinformatics Institute (EMBL-EBI), Cambridge, UK

6 Monash Institute of Pharmaceutical Sciences, Monash University, Parkville, Australia tissue typing in real time, providing a more rapid alternative to conventional histological tests during cancer surgery.

A feature of this conference was the excellent work presented by the younger members of the metabolomics community. Students and early career scientists gave over $40 \%$ of the oral presentations, and hundreds of high quality posters. Over 60 young researchers received travel awards from the U.S. National Institutes of Health (NIH), industry sponsors, academic institutions and the Metabolomics Society, making this large turnout of young scientists possible. The coveted Metabolomics Society Student Prize was awarded to Jonas Zierer (Kings College London). Jonas identified metabolic biomarkers, including gamma-glutamyl peptides and a lyso-PI lipid, that were associated with leukocyte telomere length in a large twin cohort. This study promises to open new avenues of investigation into the causes and consequences of the aging process. The inaugural Early Career award, for scientists within 5 years of completing their $\mathrm{PhD}$, was won by Andrew Palmer (EMBL, Germany). Andrew described a novel approach to annotate metabolites from MALDI imaging with high resolution MS analyses, linking biological pathways with the distribution of metabolites observed in spatial metabolomics experiments. This approach allowed significant reduction of the size of imaging MS data files while maintaining biologically interpretable information about differential metabolism between cells in a population.

\section{Biomedical metabolomics}

Metabolomics applications in the health and medical sciences continue to feature strongly, with several high quality presentations in the epidemiology, pharmacometabolomics, 
cancer, lung and cardiovascular diseases sessions. In addition, the NIH Common Fund Metabolomics Program sponsored 18 speakers for three workshops that focused on progress in biomedical and clinical applications of metabolomics, and also hosted a workshop to describe the progress of an international metabolomics ring trial. The NIH workshop presentations focused on biological investigations that were conducted with one of the six U.S. national metabolomics centers. The first workshop focused on Cancer and Lung Diseases. Through a combination of proteomics and metabolomics, Jiannong Lee (Tampa, FL) identified metabolic enzymes that are differentially regulated in small cell lung cancer tissues, consistent with six enriched metabolites (AMP, 3'-AMP, 8-Oxo-dGMP, UDPGlcNAc, Pantothenic acid and FAPy-adenine). Other highlights in this session were the discovery of metabolic differences in ovarian cancer cells when co-cultured with adipocytes, presented by Ernst Lengyel (Chicago, IL) and Ameeta Kelekar's presentation (Minneaopolis, MN) of isotope tracer experiments showing how cancer cells deal with the (toxic) free ammonia generated during glutaminolysis. Nicholas Kenyon (Davis, CA) discussed results of a double-blind, placebo-controlled, cross-over arginine intervention trial for severe asthmatic patients in which dimethyl arginine, ornithine and citrulline positively correlated with arginine plasma levels, setting the foundation to reveal patients for which such intervention may yield positive outcomes. In the second NIH workshop, Pei-an Betty Shih (San Diego, CA) proved through metabolic analysis of oxylipins that soluble epoxide hydrolases may be a novel therapeutic target for anorexia nervosa. By using NMR-based metabolomics, Laura Cox (New York, NY) found that ethanol, hepatic glutathione, maltose, and acetate were differentiating metabolites for low-dose penicillin treated mice, demonstrating a mechanism by which microbiome disruption via antibiotics early in life may increase the risk of overweight status later in childhood. Eugenia Trushina (Rochester, MN) used nontargeted and targeted metabolomic approaches to detail signatures of early energetic stress and mitochondrial dysfunction in the brain tissue of multiple transgenic animal models of Alzheimer's disease, and plasma and cerebrospinal fluid of Alzheimer's patients. The third NIH workshop focused on metabolic syndrome and kidney diseases. A highlight was the presentation by Charles Evans (Ann Arbor, MI) who used metabolomics and fluxomics to detail metabolism under exercise conditions in rats, showing that lipid and branched chain amino acid metabolism, but not glycolysis, was elevated in skeletal muscle of rats with high exercise capacity. Rhonda Cooper-deHoff (Gainesville, FL) showed that acylcarnitine levels were increased in subjects treated by the blood-pressure lowering drug atenolol, and that these metabolites marked increased insulin resistance, leading to hyperglycemia. Insulin resistance was also targeted by Brian O'Neill (Boston, MA) who used insulin-receptor knockout mice to show that disruption of the insulin/IGF-1 signaling pathway in muscle induced mitochondrial dysfunction and altered amino acid metabolism in part via autophagy-lysosomal degradation. Further studies by Patrick Brophy (Iowa City, IA) and Snezana Petrovic (Winston-Salem, NC) proved the utility of metabolomics for diagnosis of early stages of kidney diseases in neonates and adults.

\section{Plant metabolomics}

Remarkable progress has been made in plant metabolomics and related fields in the last few years, as demonstrated by a number of excellent presentations in the meeting. Two keynote lectures were devoted to plant biology. Anne Osbourn (UK) described an exciting discovery of the physical clustering of genes for some types of plant natural products, with a particular focus on triterpene diversity. Jie Luo (China) closed the conference by reporting an impressive study of the combination of crop genetics and metabolomics using metabolomic QTL and GWAS. In the session on Plant Databases and Plant Systems Biology, Mark Sumarah (Canada) reported metabolomics-assisted discovery of novel antibiotics from fungal endophytes. Ilana Rogachev (Israel) and Feng Qiu (US) described excellent methods to annotate mass spectrometry peaks, by an impressive mass spectral database of 3,300 pure natural product standards (Rogachev) and development of a plant metabolite annotation toolbox named Plant MAT (Qiu). Atsushi Fukushima (Japan) developed a metabolomic expression database on Arabidopsis thaliana named AtMetExpress. Katrin Brauner (Germany) reported a systems biology approach to uncover the diurnal regulation of sink and source interactions. In the MS imaging session, Basil Nikolau (US) presented newly developed MALDIbased MS imaging on plant tissues integrating with functional genomics analysis. Sunitha Shiva (US) was awarded two of the sponsored early-career prizes for her presentation on the identification of genes for stress-induced lipid modification by means of screening of knock-out mutants of A. thaliana. Two talks from Wolfram Weckwerth's lab (Austria) described an overview of a systems biology approach for ecological metabolomics (Weckwerth) and more specifically, molecular events in the ripening process of cacao beans by means of metabolomics and proteomics (Lei Wang). Georg Jander (US) investigated how aphid feeding impacts the metabolome and transcriptome of maize. Kris Morreel (Belgium) studied the systems biology of lignin metabolism in Arabidopsis by liquid 
chromatography-Fourier transform mass spectrometry. In the session on Compound Identification, Hiroshi Tsugawa (Japan) reported a novel strategy for MS peak annotation by means of integrated chemo- and bioinformatics resources. Immediately prior to the conference, a satellite workshop for the NSF-JST funded Metabolomics Consortium was held. Progress on technology development, plant and microbial biology, and application to a low carbon society was intensively discussed.

\section{Computational metabolomics}

This year, as before, we saw great progress in the development of computational metabolomics. A notable development at this year's meeting was a pre-conference hackathon organised by Emma Schymanski (EWAG, Ch) and Gert Wohlgemuth (UC Davis, US). The 30 participants in this workshop divided into three groups initially, discussing ideas related to data analysis workflows, data visualisation and general licensing. In the afternoon, handson work was performed on "MassBank/data exchange \& standardization", "metabolite structure identification" and "software communication". The hackathon inspired related discussions during the conference, including conversations about the "spectral hash https://github.com/ berlinguyinca/spectra-hash" and at the first open meeting of the Computational Mass Spectrometry Task Group, led by Steffen Neumann (IPB-Halle, Germany) and Sebastian Böcker (Friedrich-Schiller University, Germany).

The data processing session of the main conference program demonstrated recent examples of data processing pipelines for large scale metabolic epidemiology studies, presented by Dr Tim Ebbels (Imperial College, UK) and a series of talks on developments in imaging mass spectrometry data analysis, including applications of DESI imaging on stromal tissues in breast, colorectal and ovarian cancer. Dr Tomáš Pluskal (Okinawa Institute of Science and Technology, Japan) presented the Mass Spectrometry Development Kit (MSDK), an open-source Java library for mass spectrometry data processing, which if adopted by the community can facilitate and significantly accelerate the development of metabolomics software. Lastly, the latest advances in "in silico" prediction methods for MSMS spectra were presented by Drs Emma Schymanski, Tobias Kind, Sebastian Böcker, Ralf Weber and Robert Mistrik, with subsequent discussions on the advantages and disadvantages of each method. Finally, some new software and database tools for metabolomics were presented, including iMet by Antoni Aguilar-Mogas and MassBank of North America by Gert Wohlgemuth.

\section{Early career members network of the metabolomics society (EMN)}

The EMN provided a program of workshops and networking events to enhance the conference experience for EMN members. The workshop day kicked off with a 'Big Data' session including speakers from academia, industry, and a scientific journal. In spite of the early start, a packed room was in attendance for Prof. Douglas Kell's talk on how to handle big data sets, and what big data in metabolomics actually are. Dr Jeremy Schaver followed with an interesting talk on data visualization and how not to use statistics on large data sets. Lastly, Dr Rob Davidson from GigaScience shared his view on open access and data sharing in the metabolomics community. All in all, it highlighted the importance of Big Data and how we use it!

The EMN's next workshop, "Fluxomics, Pathway Analysis, and Metabolic Engineering for a Sustainable Future", provided interesting new perspectives on the utility of metabolomics in Metabolic Engineering. Prof. Uwe Sauer illustrated recent advances in generating hypotheses on molecular functions from metabolomics data. Prof. James C Liao talked about the development of a computational framework, termed Ensemble Modeling for Robustness Analysis (EMRA), by combining a parametricdomain integration scheme with the Ensemble Modeling approach for investigating the robustness issue of non-native pathways, namely non-oxidative glycolysis and the methanol condensation cycle. The last talk featured Prof. Akihiko Kondo who spoke on various examples of metabolic engineering for the development of microbial cell factories based on systems biology approaches.

The "Careers in Metabolomics" workshop provided an overview of several different career paths in the field of metabolomics, including government, academia, industry and a start-up company. Presenters provided advice on future directions in the field of metabolomics and tips on key strategies for early career members to develop their careers. The final workshop "Research Integrity- How to not fake your data!" covered a topic that was not only important for early career members of the Society, but for all researchers in general. Roy Goodacre, Professor of Biological Chemistry at the University of Manchester and founding editor of the Society's journal "Metabolomics" gave a great introduction to the topic by illustrating examples of scientific misconduct and how to avoid them in Metabolomics. Dr Philippe Rocca-Serra from the University of Oxford emphasized the importance of data sharing in Metabolomics whereas Dr Oliver Schmitz from Metanomics Health provided an industry perspective on research integrity. 


\section{EMN Reception at MetSoc 2015}
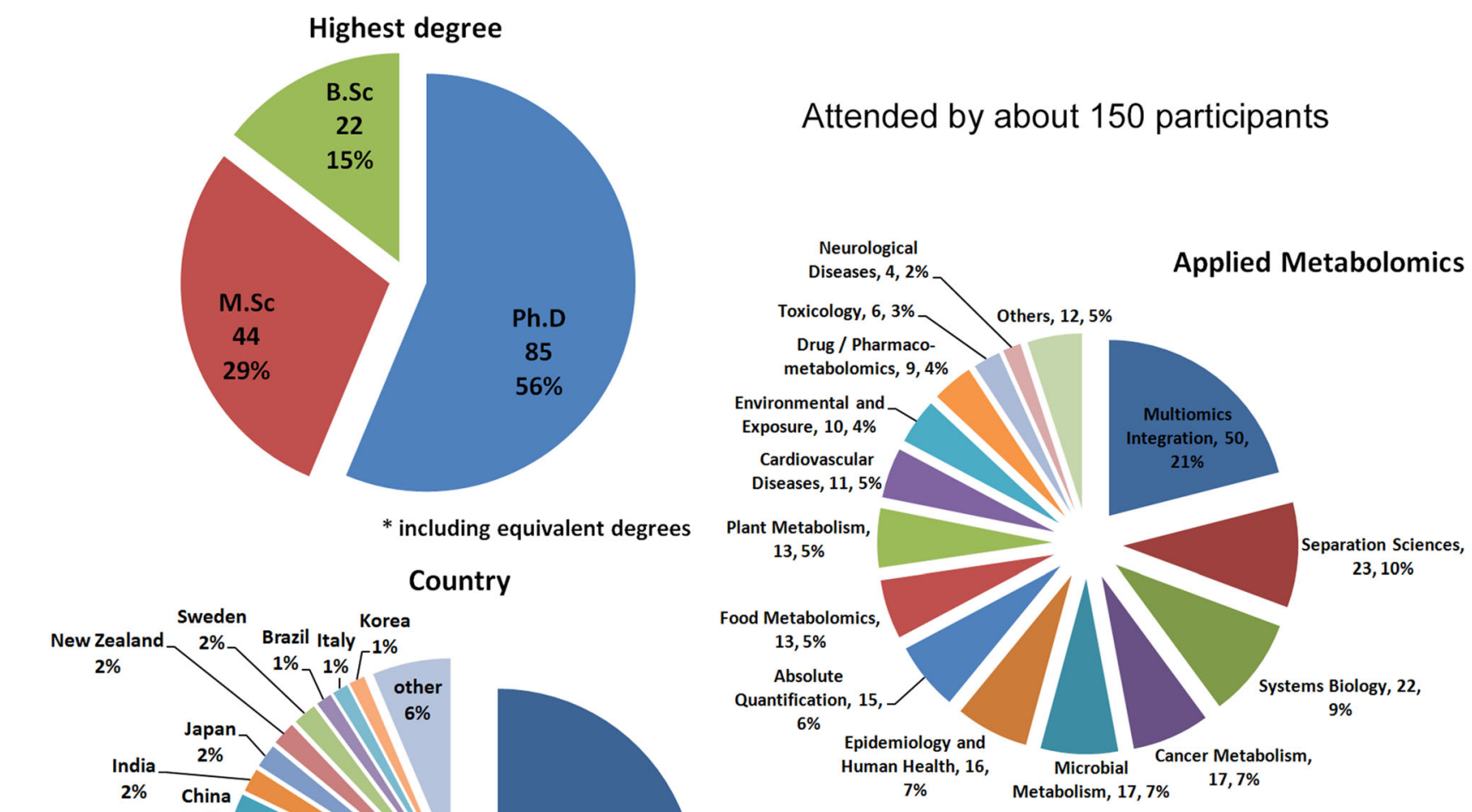

Fig. 1 Information provided voluntarily by the early career members of the Metabolomics Society attending the EMN reception in San Francisco 2015. Pie charts categorize attendees by their highest

In addition to the workshops, the EMN also hosted a pre-conference welcome reception and a group discussion featuring the Metabolomics Society's founding President, Dr Rima Kaddurah-Daouk. Rima gave a brief but comprehensive overview of the Society's history and her take on the future of metabolomics field. Both social events were well attended by over 100 early career researchers from various countries and disciplines (Figure 1).

\section{Summary and outlook}

The pre-conference workshops also provided a forum to discuss current opportunities and expectations with regards to data quality and metabolite identification, and

degree (top-left; including equivalent degrees), country (bottom-left) and interests in metabolomics applications (right; multiple answers possible)

introduced several advanced metabolomics techniques including stable isotope labelling, single cell metabolomics and novel separation capabilities. A number of new hardware, software and consumable products for metabolomics were introduced by the sponsors, and commercial providers of metabolomics services used this opportunity to demonstrate the provision of high impact and large-scale metabolomics studies. The expanded oral program allowed scientists to present new concepts in biochemistry, analytics and informatics that will further expand this growing field. A new repair biochemistry session was headlined by Andrew Hanson's description of the production and removal pathways for NAD hydrates, and a new labelling and fluxomics session included Katharina Noh's demonstration of metabolic flux analysis for improved penicillin 
production yields in an industrial setting. The full conference abstracts are available to view online (http://metabo lomics2015.org/index.php/program/full-abstract-booklet), and many of the oral presentations will be made available for download to members of the Metabolomics Society (http://metabolomicssociety.org/).

The metabolomics field continues to grow as the technology matures and diverse fields of research and industry benefit from the application of metabolomics to understand biological systems. It is expected that this trajectory will continue in the coming years as established metabolomics methods for diverse applications are now widely accessible, and new developments in hardware and software continue to emerge. As the premiere metabolomics conference series, this Metabolomics conference continued the tradition of presenting the latest advances in the development and application of metabolomics. The next international conference of the Metabolomics Society will be held in Dublin, Ireland, from 27 to 30th June 2016 (http:// metabolomics2016.org/). We hope to see you there! 